\title{
Fractional-order integral and derivative controller for temperature profile tracking
}

\author{
HYO-SUNG AHN $^{1}$, VARSHA BHAMBHANI ${ }^{2}$ and \\ YANGQUAN CHEN ${ }^{2}$
}

${ }^{1}$ Department of Mechatronics, Gwangju Institute of Science and Technology (GIST), 1 Oryong-dong, Buk-gu, Gwangju 500 712, Korea

${ }^{2}$ Center for Self-Organizing and Intelligent Systems (CSOIS), Department of Electrical and Computer Engineering, 4160 Old Main Hill, Utah State University, Logan, UT 84322-4160, USA

e-mail: hyosung@gist.ac.kr; bhambhani.v@gmail.com;

yqchen@engineering.usu.edu

MS received 15 November 2008; revised 18 February 2009

\begin{abstract}
This paper establishes a new strategy to tune a fractional order integral and derivative (ID) controller satisfying gain and phase margins based on Bode's ideal transfer function as a reference model, for a temperature profile tracking. A systematic analysis resulting in a non-linear equation relating user-defined gain and phase margins to the fractional order controller is derived. The closed-loop system designed has a feature of robustness to gain variations with step responses exhibiting a nearly iso-damping property. This paper aims to apply the analytical tuning procedure to control the heat flow systems at selected points in Quanser experimental platform. Thus, the main purpose of this paper is to examine performances of two different fractional order controllers in temperature profile tracking. From experimental comparisons with the traditional PI/PID controller based on Ziegler-Nichols' tuning method, it will be shown that the proposed methodologies are specifically beneficial in controlling temperature in time-delay heat flow systems.
\end{abstract}

Keywords. Temperature control; fractional-order integral derivative control; heat flow.

\section{Introduction}

This paper also briefly reviews fractional $M_{s}$ constrained integral gain optimization (FMIGO) (Varsha Bhambhani et al 2008), which is a proportional and integral (PI)-type control scheme, and then applies this method to temperature profile tracking. Temperature control arises in many engineering fields. For example, in cryogenic applications John et al (1985), the precision spatial temperature control via spatial heating and spatial temperature sensing is important, and in process industry (Tsai \& Lu 1998), the most common control task is to achieve 
the precise temperature profile. There are basically three types of temperature control tasks: temperature set point regulation, temperature profile tracking, and temperature uniformity control. For temperature set-point control, single loop commercial off-the-shelf temperature controllers can usually be used. Temperature profile tracking is to raise the temperature according to a prescribed temperature time history, which is required for applications such as precision heat treatment for material, batch chemical reactors, etc. The temperature uniformity control is to achieve uniformly spatially distributed temperature profile. The objective of this paper is to design a controller for temperature profile tracking at the spatially distributed places; thus, to regulate temperatures at given sensor locations to ensure that the temperatures be close to the desired values.

For temperature control, usually, On/Off control, proportional control, and traditional PID controller have been widely used (see http://www.omega.com/prodinfo/temperature controllers.html). In this paper, as a new control scheme for temperature regulation, we suggest using fractional-order ID controller for a more accurate temperature profile tracking of the spatially distributed heat flow. An actual experimental task is conducted using the heat flow equipment (HFE) of QUANSER (www.quanser.com). This paper consists of the following sections. Section 2 briefly summarizes the role of integer order PID controller in temperature control and section 3 lists basic definitions in fractional-order calculus and merits of fractional-order controller. This is followed by in depth description and derivation of proposed analytical tuning method in section 4. Then, section 5 outlines detailed descriptions of the HFE platform, system analysis, and design of a fractional controller based on the analytical tuning approach. In section 6 an extensive comparison of existing integer order solutions and fractional order solutions is presented. Finally, section 7 concludes this paper with some remarks on the achieved results and ideas for future work.

\section{Temperature control}

In temperature control, it is difficult to find the response time constant and hence on/off control scheme is usually used so as to regulate the output temperature within a dead band. However, in On/Off control, the output will be oscillatory around a set-point; so an accurate temperature profile is not achieved. For a more accurate temperature regulation, closed-loop feedback control schemes are required. Most popular control scheme is PID control, because it does not require the plant model and practically it is easily implementable. In traditional PID control, the proportional term, integral term, and derivative term have different effects on the heat flow, temperature, and speed. For temperature control, it is usually recommended to use full PID control, but with accurately-tuned-control gains. As an example of successful industrial applications, see http://www.w-dhave.inet.co.th/index/. To see various PID tuning methods for temperature control, refer to http://www.lakeshore.com/pdf_files/Appendices/LSTC _appendixF_l.pdf and "http://www.omega.com/temperature/Z/ pdf/z115-117.pdf. For a reliable temperature control, however there are some basic environmental requirements. The heater should apply enough power and temperature sensors should be spatially distributed in appropriate places. Tuning the controller means that we select the proportional, integral, and derivative gains with a particular purpose. In fact, tuning PID gains for the temperature control requires some physical interpretation about the system. It is necessary to understand the effects of the proportional, integral, and derivative terms to the system. In temperature control, individual PID gain has the following characteristics: 


\subsection{Proportional gain}

It requires more power proportional to the error between sensor temperature and the desired trajectory profile. The proportional gain is used for On/Off control. That is, when the output is within the proportional band, the power is off; but when it is out of the dead band, the power is on. If the temperature is below a set point, the output will be on longer; if the temperature is too high, the output will be off longer (http://www.omega.com/prodinfo/ temperaturecontrollers.html).

\subsection{Integral gain}

It provides a control signal that is proportional to the accumulated error. So, this integral term is for slow mode reaction and forces the steady-state error to zero for a step response. In temperature control, it adjusts the temperature to set point after stabilization.

\subsection{Derivative gain}

It provides the control force proportional to the rate of change of the output error. So, this derivative term is for fast mode reaction and yields large signal with the high-frequency control errors and with the rise or fall of system temperature.

From a literature survey, it is shown that there have been numerous applications of PID controller or fuzzy/neural network-based PID controller for the temperature control of various engineering objects. As some examples, Peter Galan showed that a fuzzy logic for enhancing PID controller is necessary for the satisfactory temperature profile tracking of injection moulding processes (http://www.manufacturing.net/ctl/article/CA408369.html) and Dihac et al (1992) used PID controller for a rapid thermal processor control. Lin et al (1999) proposed a neural fuzzy inference network for the temperature control of a water bath system and compared the performance with the PID control. Juang and Chen (2003) proposed TSK-type recurrent neural fuzzy network based on the direct inverse control configuration, which does not require a priori knowledge of the plant order and Ramos et al (2005) used PID controller to control the temperature of the bath. However, there has been no trial of using fractional-order PID controller for the temperature control of spatially distributed systems. In the next section, we briefly summarize fractional-order $\mathrm{I}^{\alpha} \mathrm{D}^{\beta}$ control and its benefits in temperature control.

\section{Fractional-order integral and derivative control}

After Newton \& Leibniz discovered calculus in the 17th century, fractional-order calculus has been studied as an alternative calculus in mathematics (Debnatho 2004). As claimed in (Chen 2004), fractional order calculus will play an important role in mechatronic and biological systems. Fractional order dynamic system and controls are relatively new research areas in control engineering. From early 90's, there has been steadily research in these areas as shown in (Lurie 1994, Podlubny 1999, Oustaloup et al 1995, Oustaloup et al 1996, Raynaud \& Zergaïnoh 2000, Vinagre \& Chen 2002, Machado 2002, Ortigueira \& Machado 2003, Lazarevic \& Debeljković 2005) even though some pioneering works can be traced back to (Manabe 1960, Manabe 1961, Oustaloup 1981, Axtell 1990). Traditional PID control method is a most popular control approach where integrator and derivative are integer order. Recently, in fractional order calculus community, a trend of using non-integer integrator or non-integer derivative for the accurate profile tracking in controlled-output has appeared, which is so-called fractionalorder PID control. In the following subsections, we briefly review this fractional-order PID control (Ahn et al 2008). 


\subsection{Fractional-order calculus}

In this paper, fractional integral is defined as:

$$
I^{\alpha} f(t)=\frac{1}{\Gamma(\alpha)} \int_{0}^{t}(t-\tau)^{\alpha-1} f(\tau) d \tau, \quad t>0, \quad \alpha \in \mathbf{R}^{+}
$$

and for the fractional derivative, Caputo derivative is used, which is defined as:

$$
D^{\alpha} f(t)=\frac{d^{\alpha} f(t)}{d t^{\alpha}}=\frac{1}{\Gamma(\alpha-n)} \int_{0}^{t} \frac{f^{(n)}(\tau)}{(t-\tau)^{\alpha+1-n}} d \tau, \quad(n-1)<\alpha \leq n
$$

where Euler's Gamma function is given as

$$
\Gamma(x)=\int_{0}^{\infty} e^{-t} t^{x-1} d t, \quad x>0
$$

with the special case when $x=n$ :

$$
\Gamma(n)=(n-1)(n-2) \cdots(2)(1)=(n-1) ! .
$$

Now, considering all the initial conditions to be zero, which is a typical assumption in fractional order derivative and fractional integral (Wen et al 2008), and using the Laplace transformation, we have $s^{-\mu} F(s)=s^{-\mu} \mathcal{L}\{f(t)\}=\mathcal{L}\left\{I^{\alpha} f(t)\right\}, \quad \mu=\alpha$, and $s^{\lambda} F(s)=s^{\lambda} \mathcal{L}\{f(t)\}=$ $\mathcal{L}\left\{\frac{d^{\lambda} f(t)}{d t^{\lambda}}\right\}$. Then, the fractional PID controller can be written as (Podlubny 1999):

$$
C(s)=K_{p}+K_{i} s^{-\mu}+K_{d} s^{\lambda} .
$$

If we take $\mu=\lambda=1$, then we obtain the classic PID controller; with $\mu=0$, it is the PD controller, and if $\lambda=0$, it is the PI controller. Also we can consider fractional order $\mathrm{I}^{\alpha} \mathrm{D}^{\beta}$ controller which is an special case of fractional PID controller with $K_{p}=0$.

\subsection{Merit of using fractional-order controller}

The idea of using fractional-order controllers for the dynamic system control is well-addressed in (Oustaloup et al 1995, Podlubny et al 1997, Oustaloup 1995). Generalized fractional-order PID controller was proposed by Podlubny et al (1996). Advantages of using fractional-order PID controller have been introduced in a number of publications. In (Monje et al 2005), it was claimed that, out of the following specifications:

(i) No steady-state error,

(ii) Phase margin and gain crossover frequency specifications,

(iii) Gain margin and phase crossover frequency specifications,

(iv) Robustness to variations in the gain of the plant,

(v) Robustness to high frequency noise,

(vi) Good output disturbance rejection, five specifications can be met by the closed-loop system, because the fractional-ordrer PID controller (4) has five tuning parameters (i.e. $\left.K_{p}, K_{i}, K_{d}, \lambda, \alpha\right)$.

In (Vinagre 2002), some observations about fractional-order PID control were given. Particularly, by varying $\alpha$ from 1 to $\infty$, it was shown that there could be a constant increment 
in the slope of the magnitude curve varying between $-20 \mathrm{db} / \mathrm{dec}$ and $0 \mathrm{db} / \mathrm{dec}$ and could be a constant delay in the phase response varying between $-\frac{\pi}{2}$ and 0 . Similarly, by changing $\lambda$ from zero to 1 , we can change the amount of phase lead and the slope of magnitude response. Vinagre et al (2000) provided frequency domain analysis to illustrate the superiority of the fractional-order PID controller applied to both the fractional dynamic system and the integer dynamic system. (Monje et al 2005) claimed that fractional-order PID controller is an adequate controller for the fractional-order mathematical models and it is less sensitive to shifts of parameters of a controlled-system and to variations of parameters of the controller. Particularly, in it was illustrated that the fractional-order PID controller is a suitable way for the control of the fractional system. Hwang et al (2002) proposed the fractionalorder band-limited compensator, which has the similar response as the fractional-order PD controller but has a less sensitivity to the high frequency noise. Leu et al (2002) provided a differential evolution algorithm to search for optimal fractional-order PID parameters to meet the phase margin and the gain margin specifications. Thus, fractional-order controller could be beneficial for temperature control since there are often variations in parameters in heat flow systems, and most of desired specifications, which are not readily achieved simultaneously by traditional PID controller, can be ensured by fractional-order closed-loop dynamics.

\section{4. $\mathrm{I}^{\alpha} \mathrm{D}^{\beta}$ gain tuning}

If the system model is given, it is easy to design a controller to control the system. However, it is not easy to design an appropriate controller for a system whose transfer function is time varying and is highly dependent on the environmental change like temperature control. Hence, it is actually quite tough to find a transfer function of the output temperature change from the power input, because the temperature of the environment is time-dependent. Furthermore, as shown in Petras \& Vinagre (2002), the transient unit response of the heat solid system could be fractional-order and as noted in (Aoki et al 2005) also, heat transfer coefficients may be fractional or non-integer order. Thus, the use of fractional-order differential model may approximate well the time-dependent behaviour of conductive systems of complex geometries with convective heat transfer. In fact, since thermal loads involve thermal diffusion, a halforder controller represents a better match to the physics of the plant to be controlled (Bohannan 2006). Therefore, for the temperature control, it is not a good idea to design a controller with fixed integer gains, and since the response of the temperature output from the power input could be fractional, the traditional integer-order controller may not provide an accurate temperature profile tracking. Thus, this paper proposes using the fractional-order $\mathrm{I}^{\alpha} \mathrm{D}^{\beta}$ controller for the accurate temperature profile tracking, which will be experimentally demonstrated by the Quanser HFE. However, note that it is very difficult to find an accurate model of transfer function of the output temperature change from the power input; thus, we use an approximated integer-order transfer function for heat flow, while a fractional-order $\mathrm{I}^{\alpha} \mathrm{D}^{\beta}$ controller is used to regulate the temperature profile. Let the plant be represented by a first order plus time delay (FOPTD) transfer function as:

$$
G(s)=\frac{K}{T s+1} e^{-L s},
$$

where $K$ is the static gain or steady state gain of the system, $L$ is the time-delay and $T$ is the time-constant of the system. These can be computed from an open loop step response of the 
system. The fractional controller is represented by $C(s)$ as:

$$
C(s)=K_{i} \frac{T s+1}{s^{\alpha}}
$$

which can be rewritten as

$$
C(s)=K_{i}\left(\frac{1}{s^{\alpha}}+T s^{1-\alpha}\right)
$$

The realization of the above fractional order controller can be done by various methods. In this research, we use a rational approximation method in implementing $s^{\alpha}$ (Podlubny et al 2002). In our implementation, we use Carlson's iteration method. Given a fractional transfer function $H(s)=(G(s))^{p}$, where $p$ is a fractional number, we define $p=1 / q$ and $m=q / 2$ in each iteration. Starting from the initial value $H_{0}(s)=1$, an approximated rational function is obtained in the form:

$$
H_{i}(s)=H_{i-1}(s) \frac{(q-m) H_{i-1}(s)^{2}+(q+m) G(s)}{(q+m) H_{i-1}(s)^{2}+(q-m) G(s)} .
$$

We use three iterations to approximate $s^{\alpha}$. The above equation (7) reveals the fractional integral derivative nature of the controller. The open-loop transfer function is given by:

$$
C(s) G(s)=K_{i} K \frac{e^{-L s}}{s^{\alpha}}, \quad 1<\alpha<2 .
$$

The gain and phase responses of the open loop transfer function are calculated as:

$$
\begin{aligned}
& |C(j \omega) G(j \omega)|=\frac{K_{i} K}{\omega^{\alpha}} \\
& \phi=-L \omega-\alpha \frac{\pi}{2} .
\end{aligned}
$$

Let us suppose that the user-defined gain margin $A_{m}$ and phase margin $\phi_{m}$, are given as:

$$
\begin{aligned}
& \left|C\left(j \omega_{p}\right) G\left(j \omega_{p}\right)\right|=\frac{1}{A_{m}} \\
& \phi_{m}=\pi+\angle C\left(j \omega_{g}\right) G\left(j \omega_{g}\right),
\end{aligned}
$$

where $\omega_{p}$ and $\omega_{g}$ are the phase and gain crossover frequencies of the open-loop system.

On substitution, the gain relations are:

$$
\begin{aligned}
\frac{K K_{i}}{\omega_{g}^{\alpha}} & =1 \\
\frac{\omega_{p}^{\alpha}}{K K_{i}} & =A_{m}
\end{aligned}
$$

and the phase relations are:

$$
\begin{aligned}
\phi_{m} & =\pi-L \omega_{g}-\alpha \frac{\pi}{2} \\
\pi & =\alpha \frac{\pi}{2}+L \omega_{p} .
\end{aligned}
$$


Therefore, we obtain:

$$
\begin{aligned}
& A_{m}=\left(\frac{\omega_{p}}{\omega_{g}}\right)^{\alpha} \\
& \frac{\omega_{p}}{\omega_{g}}=\frac{\pi-\alpha \frac{\pi}{2}}{\pi-\phi_{m}-\alpha \frac{\pi}{2}},
\end{aligned}
$$

which yields

$$
A_{m}=\left(\frac{\pi-\alpha \frac{\pi}{2}}{\pi-\phi_{m}-\alpha \frac{\pi}{2}}\right)^{\alpha} .
$$

Thus, it is shown that given gain and phase margins desired, the above equation (20) can be solved for fractional order $\alpha$ using numerical classical approach in MATLAB. However as shown in figure 1, given desired $\phi_{m}$ and $A_{m}$, there exists $\alpha$ in some restricted area. For example, if $\phi_{m}=1.4$ and $A_{m}=0.01$, then there does not exist a solution $\alpha$. Thus, there is an area that ensures existence of solutions. Therefore, we need to select $A_{m}$ and $\phi_{m}$ in an allowed area as shown in figure 1. Though this is a limitation of our new method, it reveals a better performance than existing methods as addressed in the next section. Once the value of $\alpha$ is obtained, the corresponding values of $\left(\omega_{g}, \omega_{p}, K_{i}, K_{d}\right)$ are computed as:

$$
\begin{aligned}
\omega_{g} & =\frac{\pi-\phi_{m}-\alpha \frac{\pi}{2}}{L} \\
\omega_{p} & =\frac{\pi-\alpha \frac{\pi}{2}}{L} \\
K_{i} & =\frac{\omega_{p}^{\alpha}}{K A_{m}}=\frac{\omega_{g}^{\alpha}}{K} \\
K_{d} & =T K_{i}
\end{aligned}
$$

\section{Experiment of HFE}

Quanser HFE system (see figure 2) consists of a duct equipped with a heater and a blower at one end and three temperature sensors located along the duct. The power delivered to the heater is controlled using an analog signal. For the analog signal generation and measurement, we use Quanser analog input/output libraries via MATLAB/Simulink/RTW. The fan speed of HFE is controlled using an analog signal. Fan speed is measured using a tachometer and is an input signal. Figure 2 shows the set of test equipment: computer, software, AD/DA converter and QUANSER HFE. HFE system includes built-in power module, analog signals for fan speed and power; an onboard tachometer to design speed control; and fast settling platinum temperature transducers ( 3 sensors along the duct) to measure the temperature.

This section summarizes the results of open-loop step response analysis. The fan (VF) and heater (VQ) voltages are applied in the range [0-5 Volts] and the temperature readings (degrees) at three sensor positions are recorded. The heating operation takes effect only when $\mathrm{VQ}>3 \mathrm{VDC}$. Figure 3, 4 and 5 are the open loop step response of sensor 1, 2 and 3 at varying 

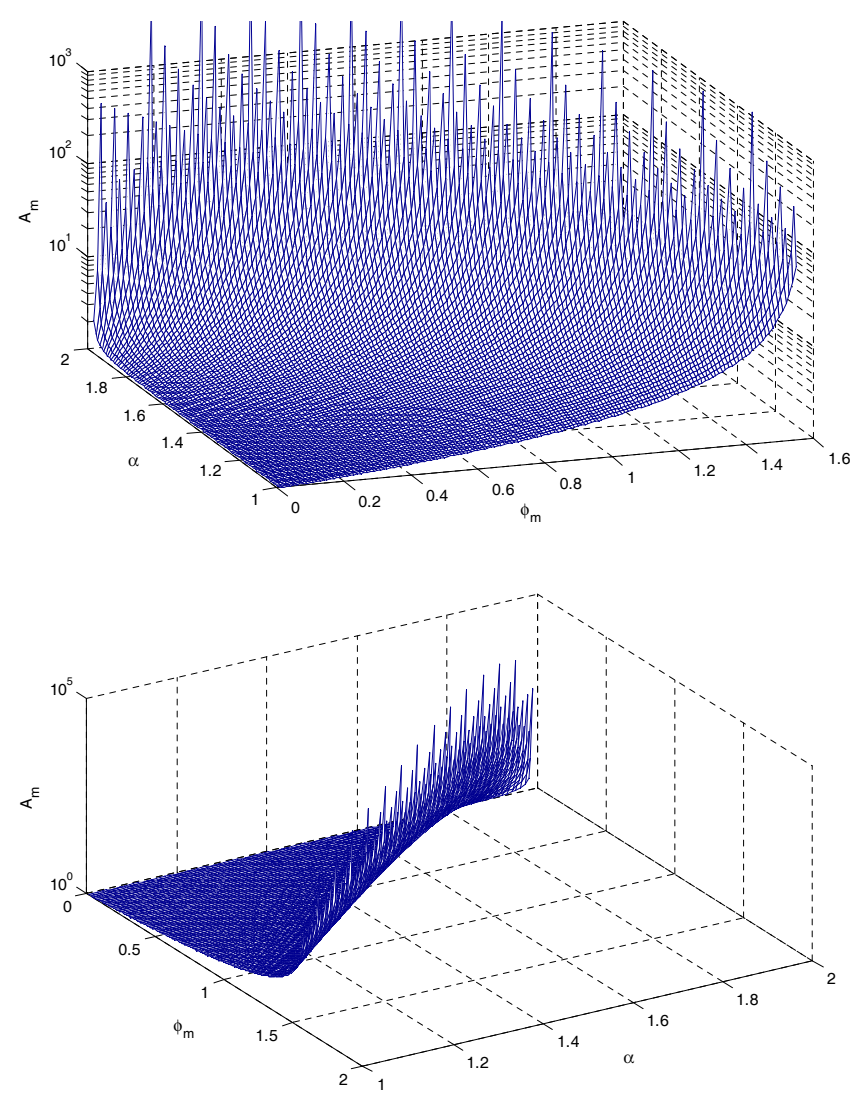

Figure 1. Relation between $A_{m}$, $\phi_{m}$, and $\alpha$ in (20).

heater (VQ) voltages but constant fan (VF) voltage of 5 VDC. The open loop response shows an initial delay, indicating that it takes a finite time for the sensors to detect the change in the temperature.

A FOPTD model at a desired sensor is given by:

$$
\frac{T_{n}}{V_{q}}=\frac{K}{T s+1} e^{-L s},
$$

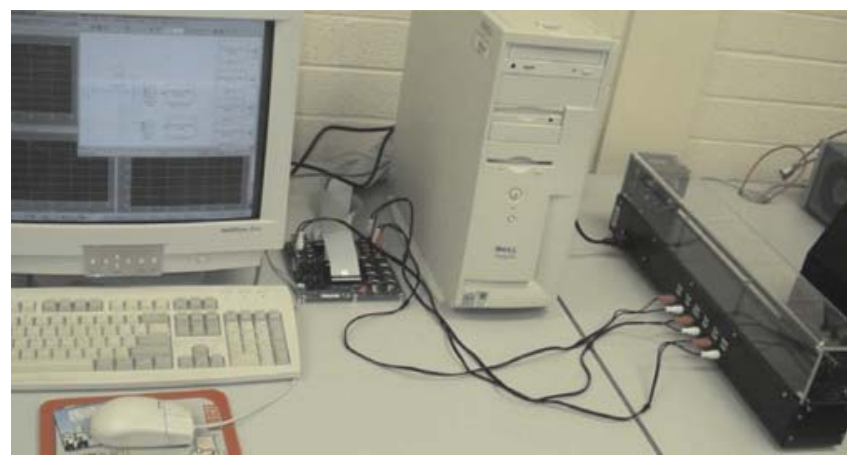

Figure 2. The set of equipment for heat flow test. 


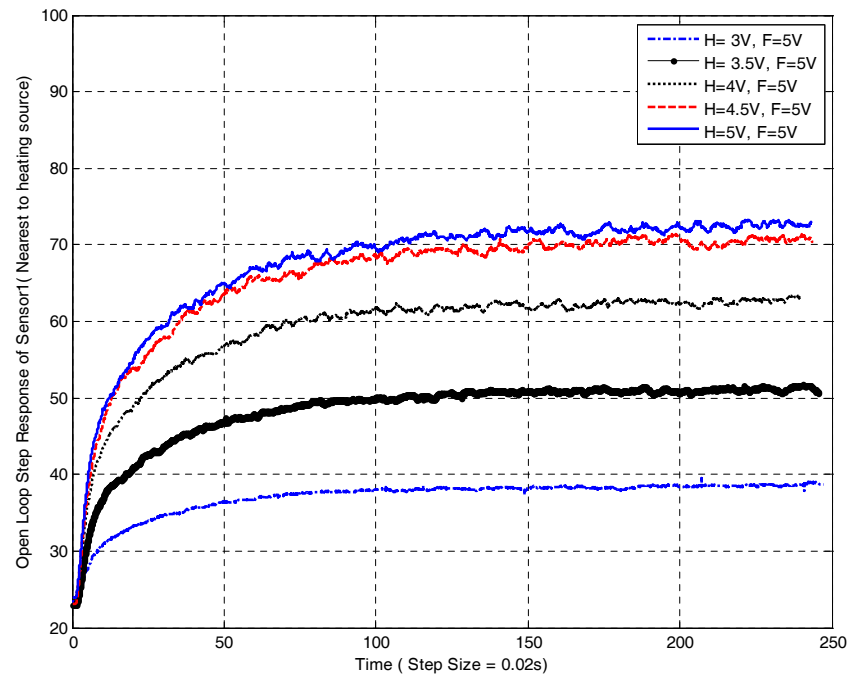

Figure 3. Sensor 1 (Fan command with constant voltage $=5 \mathrm{~V}$, Heat command with varying voltage).

where $K$ is the open loop gain given by (Max-Min)/VQ in $(\mathrm{C} / \mathrm{V})$ and Min is the temperature inside the tube before the experiment begins or the room temperature, and Max is the maximum temperature reached after 60 seconds. This is, strictly speaking, not the steady state value as the temperature is increasing. However, the rate of increase considerably slows down after 20 seconds. We now define the following parameters:

- $L$ : The delay in the response in seconds.

- $T$ : The time constant is given by the time it takes to reach $63 \%$ of the change in temperature or the time it takes for the response to reach Min $+0.63 \times(\operatorname{Max}-\operatorname{Min})$.

- $\tau$ : The relative dead time of the response given by $L /(L+T)$.

Experiments were conducted to study the open loop step response of three sensors first at constant fan voltage $(5 \mathrm{~V})$ and varying heater voltage ranging between $3-5 \mathrm{~V}$. The above

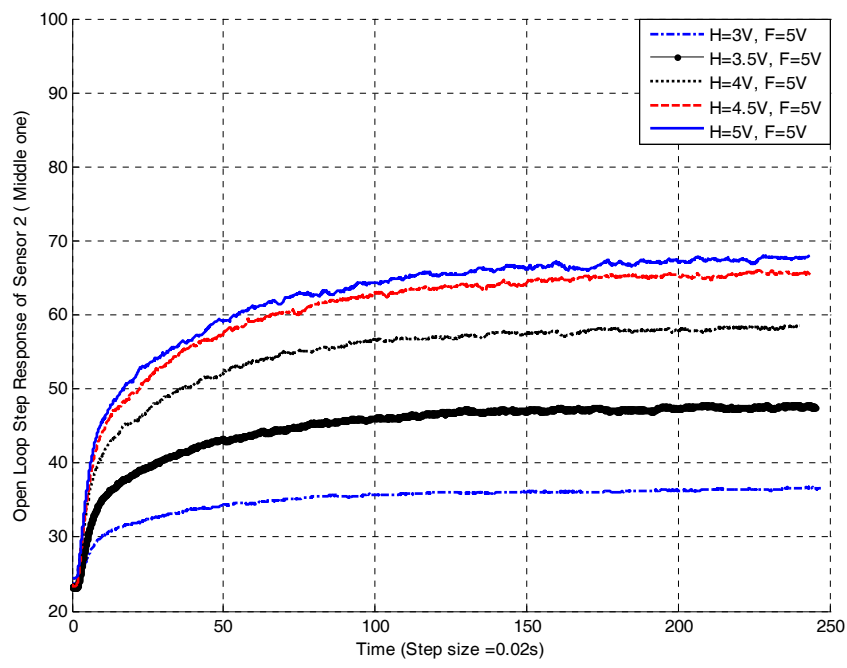

Figure 4. Sensor 2 (Fan command with constant voltage $=5 \mathrm{~V}$, Heat command with varying voltage). 


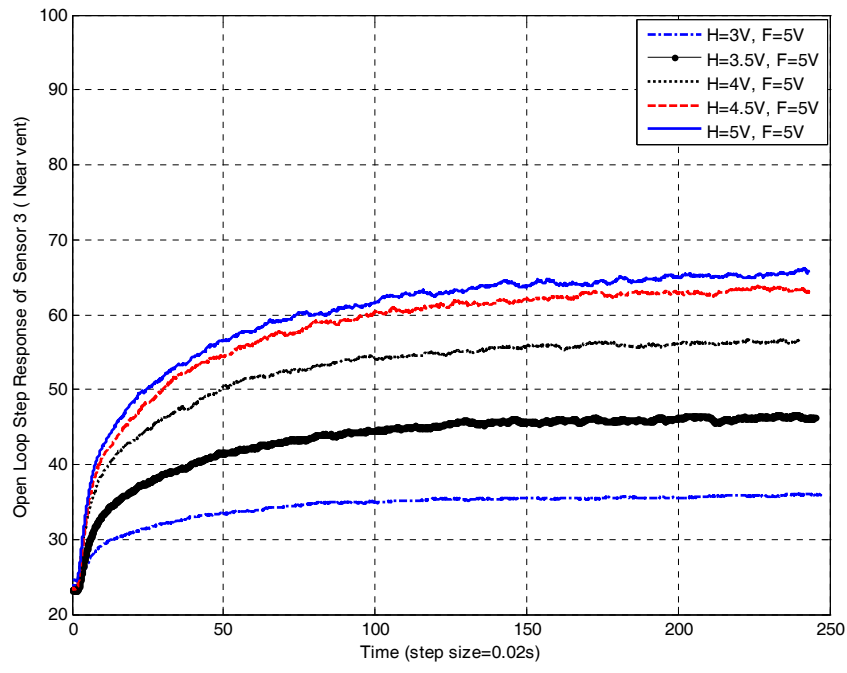

Figure 5. Sensor 3 (Fan command with constant voltage $=5 \mathrm{~V}$, Heat command with varying voltage).

experiment was repeated for constant heater voltage $(5 \mathrm{~V})$ and varying fan voltage ranging between 3 and $5 \mathrm{~V}$. The step response values obtained are listed in figure 6 . First, the heatingeffect is analyzed by keeping the fan speed constant. It is seen that as the distance of the sensors from heater increases, the corresponding gain values of the first order transfer function models of the sensors decrease and delay time increase; i.e. $K_{1}>K_{2}>K_{3}$ and $D_{1}<D_{2}<D_{3}$ where $K_{1}, K_{2}$ and $K_{3}$ are the static gain values and $D_{1}, D_{2}$ and $D_{3}$ are the delay values of the first order transfer function models of sensor 1 , sensor 2 and sensor 3 . This is shown in figures 7 and 8 . There is no trend in time constant of the three sensors with distance. However for every sensor, time constant increases as the heater voltage increases. This is shown in figure 9.

Cooling-effect is comparatively different from heating effect. This is shown in figures 10 , 11 and 12. As shown in the plots, gain values decrease as the distance from the fan increases or fan voltage increases, i.e. $K_{1}>K_{2}>K_{3}$ whereas the delay values show an increase with distance from the blower; i.e. $D_{1}<D_{2}<D_{3}$. Also there is an increase in time constant values of sensors with increase in distance from the fan, i.e. $T_{1}<T_{2}<T_{3}$. Here $T_{1}, T_{2}$ and $T_{3}$ are time constant of sensor 1 , sensor 2 and sensor 3 . Thus one can see that dynamics of HFE is really very complex and permits the need of a better controller than a simple integer

\begin{tabular}{|c|c|c|c|c|c|c|c|c|c|}
\hline \multicolumn{10}{|c|}{$\mathrm{VF}=5 \mathrm{~V}$} \\
\hline VQ & \multicolumn{3}{|c|}{ Sensor T1 } & \multicolumn{3}{|c|}{ Sensor T2 } & \multicolumn{3}{|c|}{ Sensor T3 } \\
\hline & Kp & $\mathrm{T}$ & L & Kp & $T$ & L & Kp & $\mathrm{T}$ & L \\
\hline 3 & 5.6888 & 11.36 & .36 & 4.7936 & 6.7 & .64 & 4.582 & 7.34 & .86 \\
\hline 3.5 & 8.2525 & 28.32 & .28 & 7.0526 & 22.64 & .54 & 6.6968 & 25.46 & .78 \\
\hline 4 & 9.87 & 35.22 & .18 & 8.5393 & 32.88 & .5 & 8.0449 & 38.32 & .64 \\
\hline 4.5 & 10.7428 & 47.46 & .2 & 9.4678 & 48.66 & .4 & 9.0012 & 52.38 & .7 \\
\hline 5 & 9.8883 & 46.6 & .18 & 8.7164 & 45.98 & .54 & 8.3452 & 52.38 & .66 \\
\hline \multicolumn{10}{|c|}{$V Q=5 V$} \\
\hline VF & \multicolumn{3}{|c|}{ Sensor T1 } & \multicolumn{3}{|c|}{ Sensor T2 } & \multicolumn{3}{|c|}{ Sensor T3 } \\
\hline & Kp & $\mathrm{T}$ & $\mathrm{L}$ & Кр & $T$ & L & Kp & $\mathrm{T}$ & L \\
\hline 3 & 11.2507 & 62.72 & .22 & 10.1325 & 65.74 & .5 & 9.6783 & 67.34 & .72 \\
\hline 3.5 & 10.5719 & 50.92 & .24 & 9.3853 & 51.86 & .54 & 8.858 & 53.72 & .74 \\
\hline 4 & 10.4352 & 49.52 & .14 & 9.1851 & 49.7 & .46 & 8.814 & 56.36 & .7 \\
\hline 4.5 & 10.0348 & 44.98 & .38 & 8.858 & 49.1 & .78 & 8.2915 & 50.26 & .98 \\
\hline 5 & 9.8151 & 44.84 & .22 & 8.6529 & 48.72 & .44 & 8.1353 & 48.68 & .62 \\
\hline
\end{tabular}

Figure 6. Step response data from open loop heat flow experiment. 

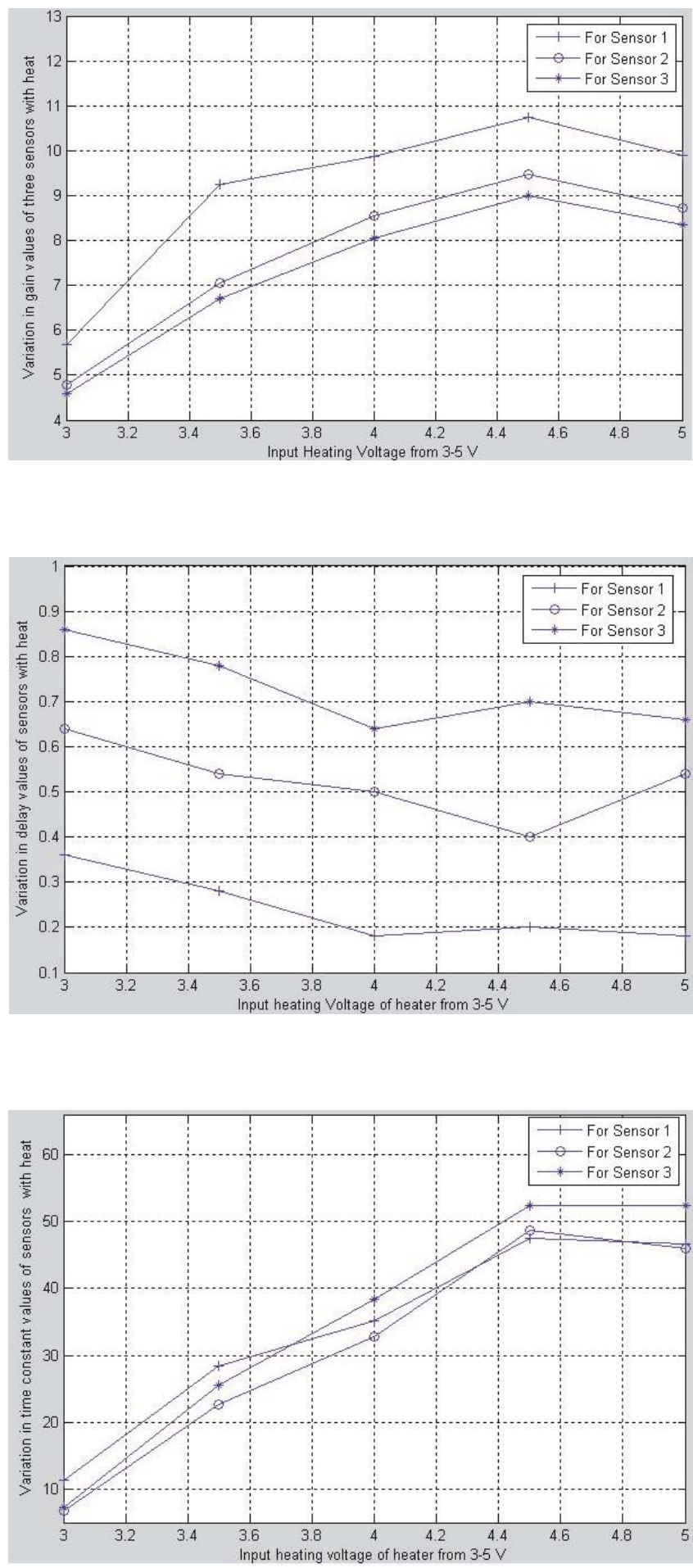

Figure 7. Heating Effect: Variation of static gain of sensors with distance.

Figure 8. Heating Effect: Variation of delay of sensors with distance.

Figure 9. Heating Effect: Time constant of sensors with distance. 

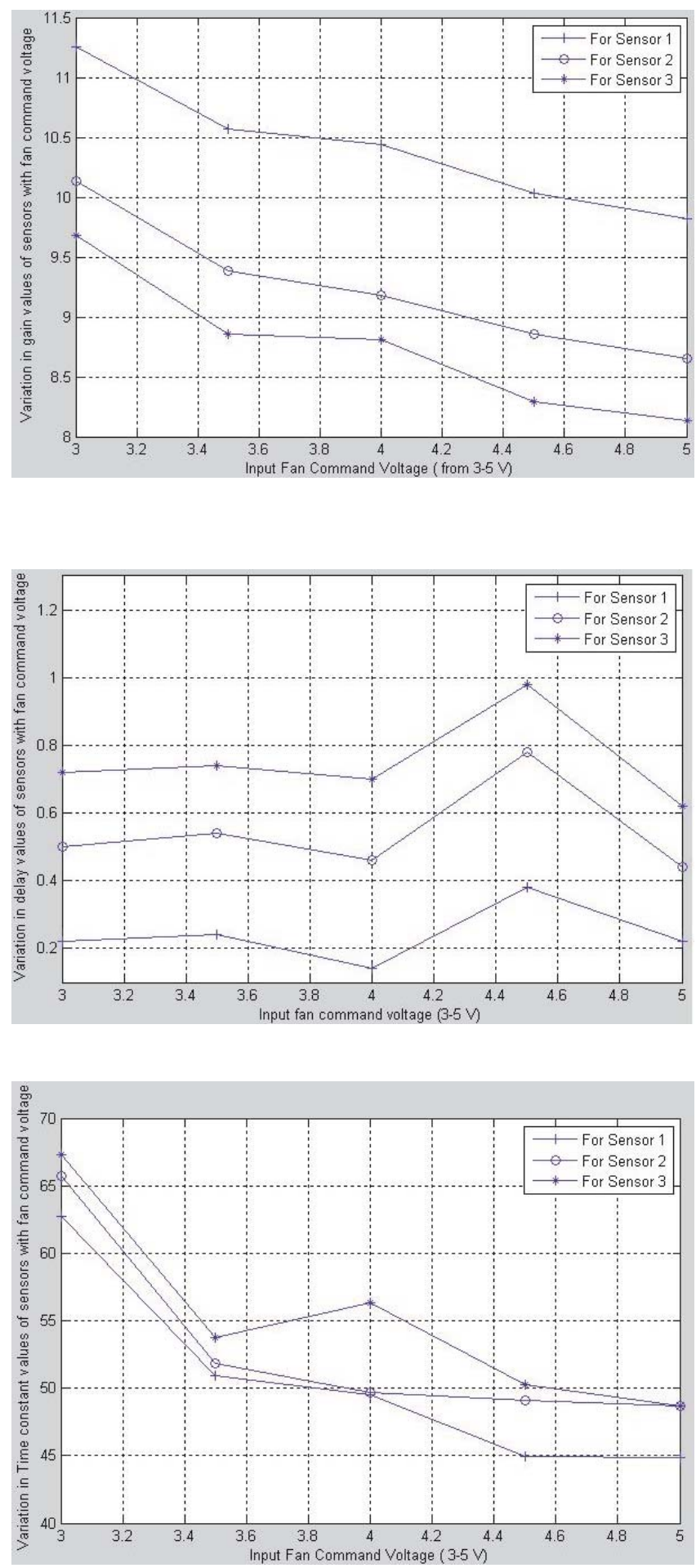

Figure 10. Cooling Effect: Variation of static gain of sensors with distance.

Figure 11. Cooling Effect: Variation of delay of sensors with distance.

Figure 12. Cooling Effect: Variation of time constant of sensors with distance. 
order PI/PID controller. Next section aims at justifying the efficiency of the new method over already existing integer and fractional order controllers.

\section{Experimental tests}

In this section, an extensive comparison of existing integer order solutions and fractional order solutions is made. For integer order case both PI and PID controllers are considered based on Ziegler-Nichols' tuning method. The Ziegler-Nichols' tuning formulae for a PI controller is given as:

$$
\begin{aligned}
& K_{p}=\frac{0.9 T}{K L} \\
& K_{i}=\frac{K_{p}}{3 L}
\end{aligned}
$$

For a PID controller the gain values are:

$$
\begin{aligned}
& K_{p}=\frac{1.2 T}{K L} \\
& K_{i}=\frac{K_{p}}{2 L} \\
& K_{d}=K_{p} \frac{L}{2} .
\end{aligned}
$$

The other controller compared in this paper is a fractional PI controller based on $M_{s}$ constrained FO-PI controller (FMIGO) tuning method (Varsha Bhambhani et al 2008) outlined as:

$$
C(s)=K_{p}^{*}+\frac{K_{i}^{*}}{s^{\alpha}}
$$

where $K_{p}^{*}$ and $K_{i}^{*}$ and $\alpha$ values are given by:

$$
\begin{gathered}
K_{p}^{*}=\frac{0.2978}{K(\tau+.000307)} \\
K_{i}^{*}=T \frac{.8578}{\tau^{2}-3.402 \tau+2.405} \\
\alpha=\left\{\begin{array}{ll}
0.7, \text { if } \tau<0.1 \\
0.9, & \text { if } 0.1 \leq \tau<0.4 \\
1.0, & \text { if } 0.4 \leq \tau<0.6 \\
1.1, & \text { if } \tau \geq 0.6
\end{array} .\right.
\end{gathered}
$$

As explained in (Bhaskaran et al 2007), FMIGO is a tuning method for fractional-order controller on the base of $M_{s}$ constrained integral optimization (MIGO), which was developed in (Astrom et al 1998; Astrom et al 2002). The motivation of MIGO is to improve upon the 


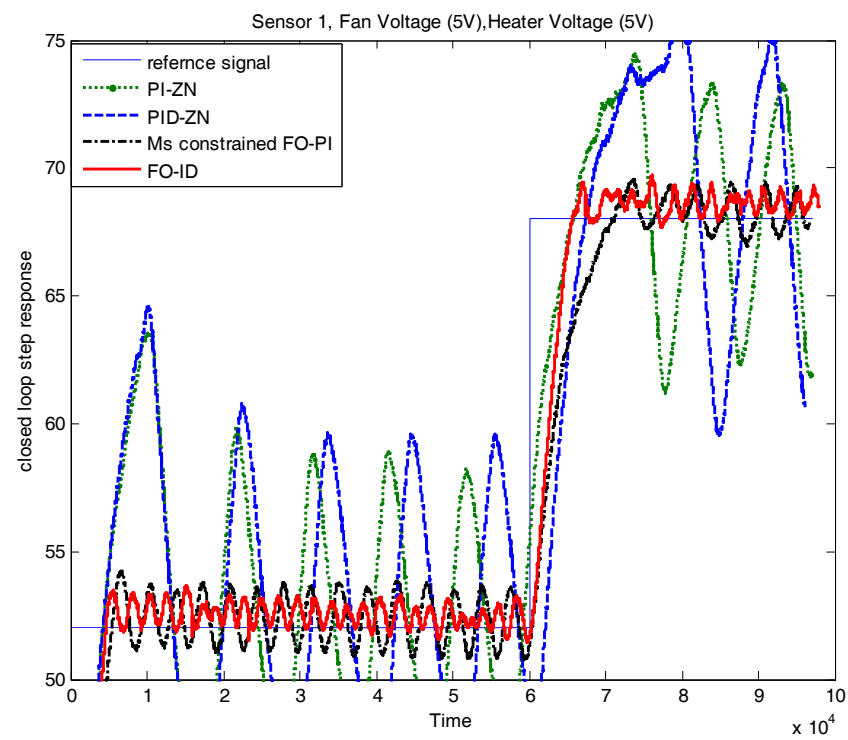

Figure 13. Controller performances for sensor 1 in real time.

Ziegler-Nichols' tuning rules to overcome two major drawbacks: (i) very little process information was taken into account as the rules were based on the two parameter characterization of the system dynamics based on step response data and (ii) the quarter amplitude damping design method exhibits very poor robustness. To overcome these drawbacks, in (Astrom et al 1998; Astrom et al 2002), a new criterion for developing tuning method for the PI controllers based on robust loop shaping is employed. The FMIGO is a generalized version of MIGO method aiming at obtaining the gains of the $\mathrm{PI}^{\alpha}$ at any given fractional order $\alpha$ (Varsha Bhambhani et al 2008).

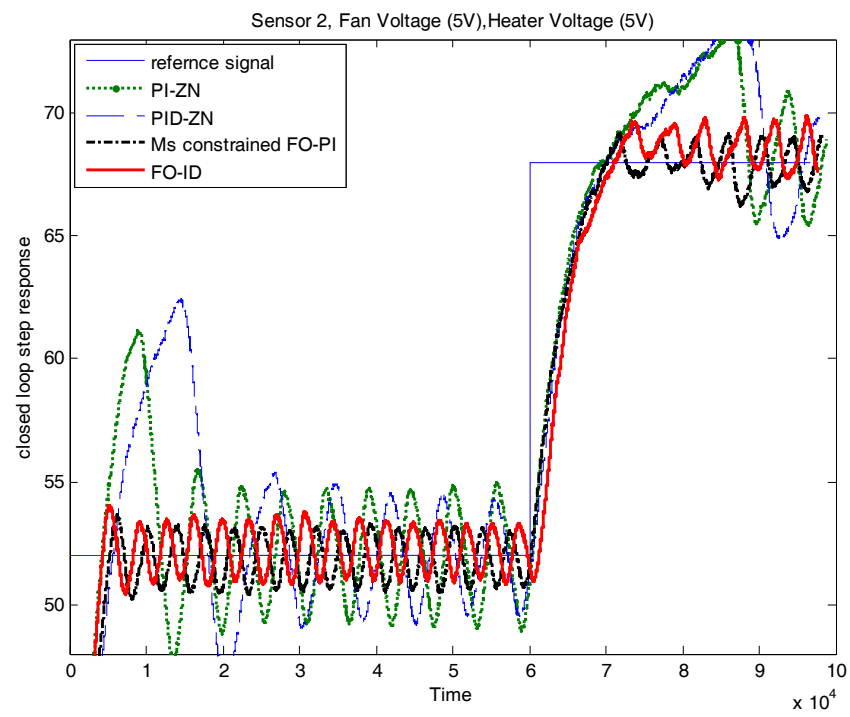

Figure 14. Controller performances for sensor 2 in real time. 


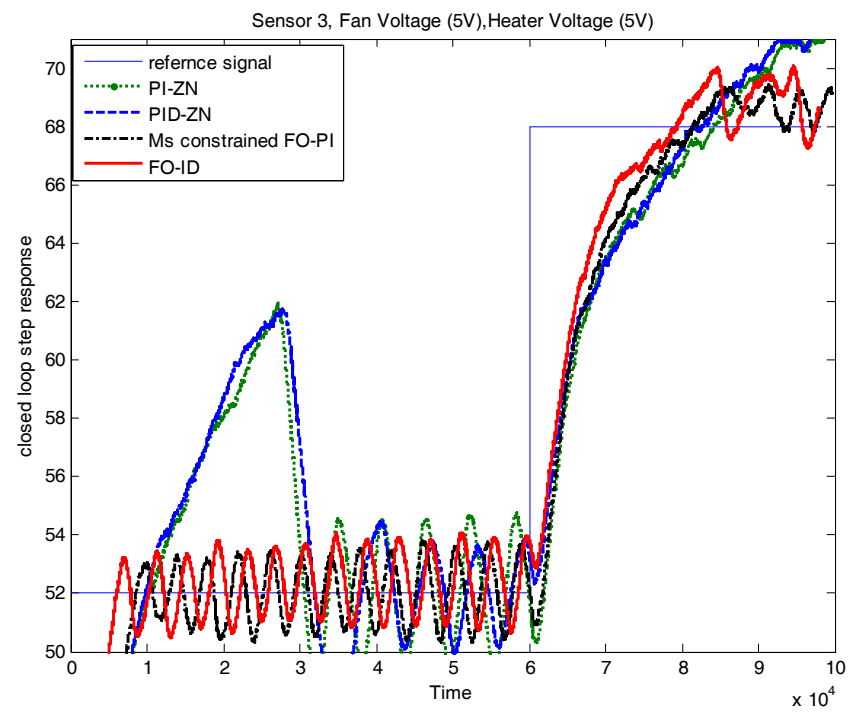

Figure 15. Controller performances for sensor 3 in real time.

The controller performances for all the three sensors are compared in real time as shown in figures 13, 14 and 15. As is clear from the figures, the fractional controllers outperform simple integer order PI/PID controller. The new controller (fractional ID controller) is tuned better in comparison to $M_{s}$ constrained FO-PI controller (FMIGO) for sensor 1 whereas for sensor 2 and sensor 3 both the fractional order controllers perform more or less the same. The same results in MATLAB Simulink environment are shown in figure 16, which is the controller performances for sensor1. The simulation and actual lab results showed some mismatchings indicating that the linear model was unable to model the system nonlinearities. The

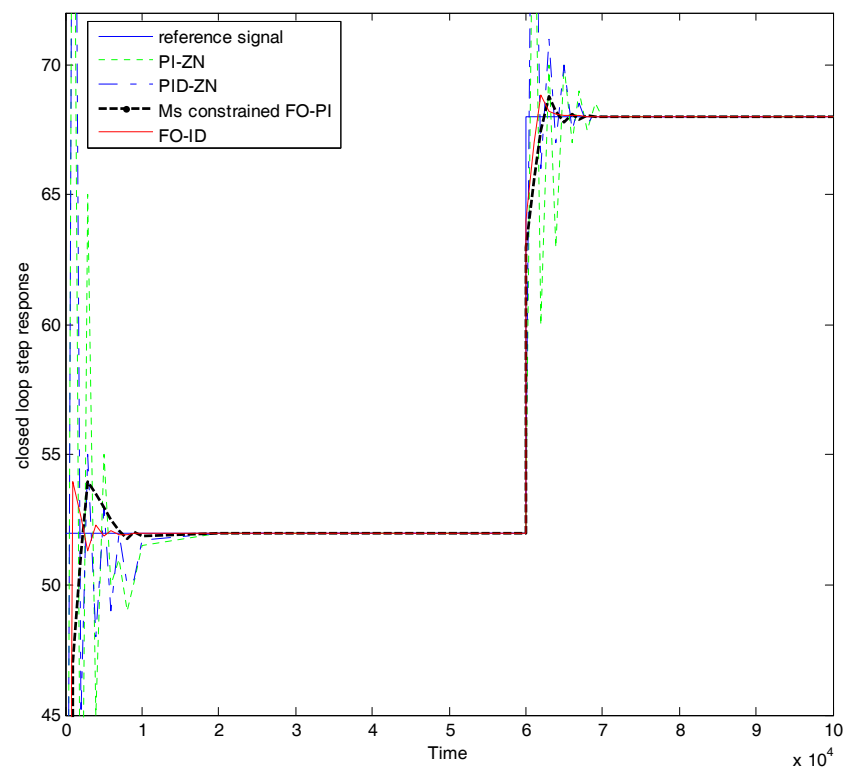

Figure 16. Controller performances for sensor1 in Simulink. 
discrepancy between simulation and experimental results may be due to employing integerorder model of the heat flow (5) for tuning fractional-order controller (7). It is noticeable that fractional order controllers could be regarded as interpolations of conventional PI, PD, and PID controllers. Fuzzy and neural network controllers may have a similar effect as fractional order controllers. However, fuzzy PID controllers or neural network PID controllers try to find optimal control gains using input and output mappings (Malki et al 1997, Tang et al 2001, Wang et al 2007). Thus, though fuzzy PID and neural network PID could be considered as optimal tuning methods of traditional integer order controllers when the control structure is fixed, they cannot represent fractional order effects.

\section{Conclusions}

The objective of this paper is to introduce a novel analytical tuning method for a fractional integral derivative $\left(\mathrm{I}^{\alpha} \mathrm{D}^{\beta}\right)$ controller (FO-ID) and apply the results to analyse the heat flow in HFE module. The performance of new FO-ID controller was compared to the integer-order PI/PID controller and FO-PI controller. Our observation is that since the HFE systems have very small relative dead time, a full integrator is not necessary. The FO-PI controller gives a strong competition to FO-ID controller tuned by new analytical method for some cases. For FO-ID method introduced, the resulting closed loop system has the desirable feature of being robust to gain variations with step responses exhibiting a nearly iso-damping property. Extensive simulation results are included to illustrate the simple yet practical nature of the developed new tuning rules. However as admitted in the previous section, there is a discrepancy between simulation and experimental results due to inaccurate modelling of the temperature system. In our future research efforts, we would like to consider a model uncertainty problem by employing a robust fractional order controller, which is to be researched further in this field.

The reviewers' comments, which have improved the quality of this paper, are greatly appreciated. The research of this paper was supported in part by Korea Science and Engineering Foundation (KOSEF, Project No. 2009-0071105) and in part by a grant from the Institute of Medical System Engineering (IMSE) in the GIST.

\section{References}

Ahn Hyo-Sung, Varsha Bhambhani, Chen YangQuan 2008 Fractional-order integral and derivative controller design for temperature profile control. In Proc. of the 2008 Chinese Control and Decision Conference 1-6

Aoki Yoshinori, Sen Mihir, Paolucci Samuel 2005 Approximation of transient temperatures in complex geometries using fractional derivatives. Technical note, Department of Aerospace and Mechanical Engineering, University of Notre Dame, Notre, IN

Åström K, Panagopoulos H, Hägglund T 1998 Design of PI controllers based on non-convex optimization. Automatica 34: 585-601

Åström K, Panagopoulos H, Hägglund T 2002 Design of PID controllers based on constrained optimization. IEE Proceedings of Control Theory and Application 149: 32-40

Axtell M, Bise E M 1990 Fractional calculus applications in control systems. In Proceedings of the IEEE 1990 Nat. Aerospace and Electronics Conf., New York, USA 563-566 
Bhaskaran T, Chen Y Q, Xue D 2007 Practical tuning of fractional order proportional and integral controller (1): Tuning rule development. In Proceedings of ASME 2007 International Design Engineering Technical Conferences \& Computers and Information in Engineering Conference, IDETC/CIE 2007, Las Vegas, NV, USA 1-12

Bohannan Gary W 2006 Analog fractional order controller in a temperature control application. In 2006 IFAC Workshop on Fractional-order Control 1-6

Chen YangQuan, Xue Dingyü 2004 Huifang Dou Fractional calculus and biomimetic controls. In Proc. of the First IEEE Int. Conf. on Robotics and Biomimetics (RoBio04), Shengyang, China 901-906

Debnatho L 2004 A brief historical introduction to fractional calculus. Int. J. Math. Educ. Sci. Technol. 35: 487-501

Dilhac J-M, Ganibal C, Bordeneuve J, Nolhier N 1992 Temperature control in a rapid thermal processor. IEEE Transactions on Electron Devices 39: 201-203

Hwang Chyi, Leu Jeng-Fan, Tsay Sun-Yuan 2002 A note on time-domain simulation of feedback fractional systems. IEEE Transactions on Automatic Control 47(4): 625-631

John M Swartz, Lawrence G Rubin Fundamentals for usage of cryogenic temperature controllers. Lake shore cryotrnics application note, Lake Shore Cryotrnoics, Westerville, OH

Juang Chia-Feng, Chen Jung-Shing 2003 A recurrent neural fuzzy network controller for a temperature control system. In Proc. of the 12th IEEE International Conference on Fuzzy Systems 408-413

Lazarević M P, Debeljković Lj D 2005 Finite time stability analysis of linear autonomous fractional order systems with delayed state. Asian J. Control 7(4): 440-447

Leu Jeng-Fan, Tsay Sun-Yuan, Hwang Chyi 2002 Design of optimal fractional-order pid controllers. J. Chin. Inst. Chem. Engrs. 33: 193-202

Lin Chin-Teng, Juang Chia-Feng, Li Chung-Ping 1999 Temperature control with a neural fuzzy inference network. IEEE Transactions on Systems, Man and Cybernetics, Part C 29: 440-451

Lurie Boris J 1994 Three-parameter tunable tilt-integral-derivative (TID) controller. US Patent US 5371670

Machado Tenreiro J A 2002 Special issue on fractional calculus and applications. Nonlinear Dynamics (Guest Editor) 29: 1-385

Malki Heidar A, Dave Misir, Denny Feigenspan, Guanrong Chen 1997 Fuzzy PID control of a flexiblejoint robot arm with uncertainties from time-varying loads. IEEE Trans. on Control Systems Tech. 5: $371-378$

Manabe S 1960 The non-integer integral and its application to control systems. JIEE (Japanese Institute of Electrical Engineers) Journal 80(860): 589-597

Manabe S 1961 The non-integer integral and its application to control systems. ETJ of Japan 6(3-4): 83-87

Monje C A, Vinagre B M, Chen Y Q, Feliu V, Lanusse P, Sabatier J 2005 Book chapter in fractional derivatives and their applications. Part 3: Systems analysis, implementation and simulation, systems identification and control, UBooks, Augsburg, Germany

Ortigueira Manuel Duarte, Machado Tenreiro J A 2003 Special issue on fractional signal processing and applications. Signal Processing (Guest Editors) 83(11) 2285-2480

Oustaloup A 1981 Fractional order sinusoidal oscilators: Optimization and their use in highly linear FM modulators. IEEE Transactions on Circuits and Systems 28(10): 1007-1009

Oustaloup A 1995 La Dérivation non Entière, HERMES, Paris

Oustaloup A, Mathieu B, Lanusse P 1995 The CRONE control of resonant plants: application to a flexible transmission. European Journal of Control 1(2):

Oustaloup A, Moreau X, Nouillant M 1996 The CRONE suspension. Control Engineering Practice 4(8): 1101-1108

Petras I, Vinagre B M 2002 Practical application of digital fractional-order controller to temperature control. Acta Montanistica Slovaka 7(2): 131-137

Podlubny Igor, Ahmed M A 1996 El-Sayed On two definitions of fractional derivatives. Tech. Rep. UEF-03-96, Slovak Academy of Sciences. Institute of Experimental Physics, Department of Contrlo Engineering. Faculty of Mining, University of Tecnology. Kosice 
Podlubny I, Dorcak L, Kostial I 1997 On fractional derivatives, fractional-order dynamic systems and $p i^{\lambda} d^{\mu}$-controllers. In Proc. of the 36the Conference on Decision and Control, San Diego, CA 4985-4990

Podlubny Igor 1999 Fractional-order systems and $\mathrm{PI}^{\lambda} \mathrm{D}^{\mu}$-controllers. IEEE Trans. Automatic Control 44(1): 208-214

Podlubny I, Petráš I, Vinagre B M, Đleary P, Dorčák L' 2002 Analogue realizations of fractional-order controllers. Nonlinear Dynamics 29(1-4): 281-296

Ramos H M S G, Assuncao F, Ribeiro A L, Ramos P M 2005 A low-cost temperature controlled system to test and characterize sensors. In Proc. of the 7th AFRICON Conference in Africa 457-460

Raynaud H F and Zergainoh A 2000 State-space representation for fractional order controllers. Automatica 36: 1017-1021

Tang K S, Man Kim Fung, Chen Guanrong, Kwong Sam 2001 An optimal fuzzy PID controller. IEEE Trans. on Industrial Electronics 48: 757-765

Tsai Ching-Chih, Lu Chi-Huang 1998 Multivariable self-tuning temperature control for plastic injectionmolding process. IEEE Transactions on Industry Applications 34: 310-318

Varsha Bhambhani, Chen YangQuan, Xue Dingyu 2008 Optimal fractional order proportional integral controller for varying time-delay systems. In Proc. of the 17th IFAC World Congress Seoul, Korea, IFAC 4910-4915

Vinagre Blas M 2002 Fractional order systems and fractional order control actions, http://mechatronics. ece.usu.edu /foc/cc02tw/cdrom/lectures/book.pdf Las Vegas, NE, USA

Vinagre Blas M, Chen YangQuan 2002 Lecture notes on fractional calculus applications in automatic control and robotics. In the 41st IEEE CDC2002 Tutorial Workshop \# 2, Vinagre Blas M, Chen YangQuan (eds.) Las Vegas, Nevada, USA 1-310 [Online] http://mechatronics.ece.usu.edu /foc/cdc02_tw2_ln.pdf.

Vinagre B M, Podlubny I, Dorcak L, Feliu V 2000 On fractional PID controllers: A frequency domain approach. In Proc. of the IFAC Workshop on Digial Control: Past, Present and Future of PID control, Terrassa, Spain

Xiang-Jun Wen, Zheng-Mao Wu, Lu Jun-Guo 2008 Stability analysis of a class of nonlinear fractionalorder systems. IEEE Trans. on Cicruits and Systems II: Express Briefs 55: 1178-1182

Wang Jiangjiang, Zhang Chunfa, Jing Youyin, Dawei 2007 An Study of neural network PID control in variablefrequency air-conditioning system. In Proc. of the IEEE International Conference on Control and Automation, Guangzhou, China 317-322 